Check for updates

Cite this: RSC Adv., 2017, 7, 44124

\title{
miRNA-21 promotes renal carcinoma cell invasion in a microfluidic device
}

\author{
Xiaogang Wang, (D) $\dagger^{\mathrm{a}}$ Yiying Jin, $\dagger^{\mathrm{a}}$ Wei Wang, ${ }^{\mathrm{a}} \mathrm{Na} \mathrm{Yu},{ }^{a}$ Xue Xiao, ${ }^{\mathrm{c}}$ Tingjiao Liu ${ }^{* b}$ \\ and Xiancheng Li ${ }^{\star a}$
}

MicroRNAs (miR) play important roles in cancer invasion. In this study, we investigated the role of miR-21 in the invasion of renal cell carcinoma. The invasive abilities of renal carcinoma cell lines (786-O, Caki-1, A498), one normal epithelial kidney cell line (HK-2) and primary renal carcinoma cells were assessed in a microfluidic model. The expression of miR-21 was examined by quantitative real time PCR (qRT-PCR). We found that HK-2 cells with low miR-21 expression showed low invasive ability while renal carcinoma cell lines with high miR-21 expression showed high invasive ability. Primary renal cancer cells showed higher invasive ability than HK-2 cells, and this was consistent with the level of miR-21 expression. miR21 knockdown reduced both renal carcinoma cell line and primary cancer cell invasion. Overall, this study shows that miR-21 promotes renal cancer cell invasion in both cell lines and primary cancer cells and suggests that miR-21 might be a new target in renal cancer therapy. In addition, we demonstrate that a microfluidic device provides a useful in vitro platform to study cancer cell invasion.

Received 12th February 2017 Accepted 29th July 2017

DOI: 10.1039/c7ra01768e

rsc.li/rsc-advances microchannels, cell invasion can be observed in the microfluidic systems. ${ }^{\mathbf{1 4 - 1 6}}$ For example, a microfluidic device was developed for monitoring cell migration across extracellular matrix-coated microgaps (ECM). ${ }^{\mathbf{1 4}}$ Migration of invasive MDAMB-231 cells was tracked by real-time light microscopy. Similarly, transendothelial invasion of tumor aggregates was successfully observed in a microfluidic system. ${ }^{16}$ For these reasons, the use of a microfluidic device provides a favorable method of studying the processes of RCC invasion and metastasis.

Although there has been widespread research into genetic biomarkers for RCC, epigenetic biomarkers including microRNAs (miR) have also received considerable attention because of their biological and clinical utility in diagnosis and treatment, especially miR-21. ${ }^{17}$ miR-21 was one of the first oncomiRs to be identified, and it has since been confirmed to be upregulated in numerous types of human cancers, including RCC. ${ }^{18}$ Previous studies have shown that miR-21 plays an important role in both RCC growth and invasion. ${ }^{\mathbf{1 9 , 2 0}}$ Studies have shown that the higher the level of expression of miR-21, the greater the invasive ability of RCC cells, ${ }^{21,22}$ and at least two studies showed that elevated expression of miR-21 was associated with poor survival in RCC patients. ${ }^{23}$ miR-21 and its target programmed cell death 4 (PDCD4) will provide a strong candidate for therapeutic applications in renal cancer in the future. ${ }^{24,25}$ That miR21 plays an important role in renal cell carcinomas has been proposed based on profiling studies using renal carcinoma cell lines and tumor tissue samples. However, the function of miR21 in causing primary renal cancer cell invasion has rarely been shown. We used primary renal cancer cell and microfluidic
${ }^{a}$ Department of Urology, The Second Affiliated Hospital of Dalian Medical University, Dalian, Liaoning, China.E-mail: xiancheng_li@sina.cn

${ }^{b}$ Section of Oral Pathology, College of Stomatology, Dalian Medical University, Dalian, Liaoning, China

'Dalian Medical University, Dalian, Liaoning, China.E-mail: tingjiao@dlmedu.edu.cn $\dagger$ Co-first authors. 
model to mimic environment in body, and observed cancer cell invasion directly. The aims of this study were to use a microfluidics device to observe the invasion of RCC at the primary cellular level and to investigate whether knockdown of miR-21 could reduce the invasive ability of RCC.

\section{Materials and methods}

\section{General cell culture and primary cell isolation}

Our human cell lines (786-O, Caki-1, A498 and HK-2) were obtained from the Institute of Biochemistry and Cell Biology of the Chinese Academy of Sciences (Shanghai, China) as a commercial source. Normal renal HK-2 cells were cultured as a monolayer in Dulbecco's Modified Eagle Medium: nutrient mixture F12 (DMEM/F12; Hyclone, Logan, UT, USA) with 10\% fetal bovine serum (FBS; ScienCell, Carlsbad, CA, USA), $100 \mathrm{U} \mathrm{mL}^{-1}$ penicillin and $100 \mathrm{U} \mathrm{mL}^{-1}$ streptomycin (Hyclone). The RCC cell lines were maintained in RPMI 1640 medium supplemented with $10 \%$ FBS (ScienCell), $100 \mathrm{U} \mathrm{mL}^{-1}$ penicillin and $100 \mathrm{U} \mathrm{mL}^{-1}$ streptomycin (Hyclone). All cells lines were maintained in an incubator with a humidified atmosphere of $95 \%$ air and $5 \% \mathrm{CO}_{2}$ at $37{ }^{\circ} \mathrm{C}$.

Primary tumor cells were isolated from fresh RCC samples. This study was performed in strict accordance with the NIH guidelines for the care and use of laboratory animals (NIH Publication No. 85-23 Rev. 1985) and was approved by the Institutional Animal Care and Use Committee of National Tissue Engineering Center (Shanghai, China). The study protocol was approved by the Ethics Committee of the Second Affiliated Hospital of Dalian Medical University, all experiments were performed in compliance with relevant laws or guidelines and followed institutional guidelines. And we have informed consent of the patient was obtained. The patients did not receive any chemotherapy or radiotherapy before surgery. The tissues were minced and digested with collagenase IV (Invitrogen, Carlsbad, CA, USA) for $1 \mathrm{~h}$ at $37^{\circ} \mathrm{C}$ with shaking. The dissociated tissues were then incubated without shaking for $5 \mathrm{~min}$ at room temperature, followed by separation of the supernatant to a new tube and centrifugation (1000 rpm, $5 \mathrm{~min})$. The cell pellet was resuspended in fresh culture medium and incubated at $37^{\circ} \mathrm{C}$ with $5 \% \mathrm{CO}_{2}$ and $95 \%$ relative humidity.

\section{Chip fabrication and cell invasion assay using a microfluidic model}

We constructed a device composed of two layers: the polydimethylsiloxane (PDMS) layer and a glass substrate. The PDMS layer was fabricated by replicate molding of the master, which was prepared by spin-coating SU8-2035 negative photoresist (Microchem Corp., Westborough, MA, USA) onto a glass wafer, and then patterned by photolithography. Double coating and patterning SU8-2035, respectively, led to the formation of microstructures with heights of 100 and $50 \mu \mathrm{m}$. The lower microstructure was defined by patterning the first layer of SU82035 with a thickness of $100 \mu \mathrm{m}$. The second $50 \mu \mathrm{m}$ thickness SU8-2035 layer was then patterned to form the higher microstructure. Sylgard 184 PDMS base and curing agent (Sylgard
Silicone elastomer 184, Dow Corning Corp., Midland, MI, USA) were mixed thoroughly (10:1 by mass), degassed under vacuum, and poured onto the master. The polymer curing process was conducted in an oven for $1 \mathrm{~h}$ at $80^{\circ} \mathrm{C}$. After cooling, the PDMS layer was gently peeled off the master and trimmed to size. Inlet and outlet holes were created by punching through the PDMS with a razor-sharp punch. The piece of PDMS was irreversibly bonded to a glass slide after oxygen plasma treatment for $60 \mathrm{~s}$. Prior to use, the device was sterilized with UV light for $30 \mathrm{~min}$.

To evaluate the invasive potential of RCC, a microfluidic device developed in our previous study was used. Matrigel ${ }^{\mathrm{TM}}$ (Corning), as the substitute for ECM, was loaded into the matrix inlet. Matrigel was thawed at $4{ }^{\circ} \mathrm{C}$ in a refrigerator overnight. Next, the cold liquid state Matrigel was loaded into the matrix inlet of the microfluidic device. Driven by capillary force, the liquid flowed into stimulation channels and arrived at the junction between the main channel and side channels. Rapid enlargement of the height of the main channel changes the physical angle between the liquid interface and solid surface; hence, a temporary barrier for the advancing liquid is formed. Thus, Matrigel stopped at the interface of the main channel and side channels. Matrigel gelled in less than $30 \mathrm{~min}$ at room temperature. To assess the invasive activity of 786-O, Caki-1, A498, HK-2 and primary RCC cells, the cells were seeded into the cell culture channel in serum-free medium, the density of cell was $1 \times 10^{6} \mathrm{~mL}^{-1}$, and medium with $20 \%$ FBS was loaded into the stimulation channel. The cell invasion area was determined as the area invaded by cells in the matrix channel and measured by Image-Pro Plus 6.0 (Media Cybernetics, Rockville, MD, USA). The data represent at least three experiments performed in triplicate (mean \pm standard error).

\section{Cell invasion assay using Transwell ${ }^{\circledR}$ plates}

Transwell chambers (Corning) pre-coated with Matrigel (Corning) were used to perform the Transwell assay, evaluating cell invasion. The cell suspension $\left(1 \times 10^{6}\right.$ cells per $\left.\mathrm{mL}\right)$ was prepared in 1640, following which $200 \mu \mathrm{L}$ cell suspension was added into the upper chamber, and $600 \mu \mathrm{L} 1640$ supplemented with $20 \%$ FBS was added into the lower chamber. After incubating for $24 \mathrm{~h}$ at $37^{\circ} \mathrm{C}$ in $5 \% \mathrm{CO}_{2}$, the medium in the chambers was removed, the cells were fixed in $4 \%$ paraformaldehyde for 20 min, washed three times with PBS, and stained with $0.1 \%$ crystal violet (Solarbio, Beijing, China) for $20 \mathrm{~min}$. The cells adhering to the upper surface of the membrane were removed using a cotton applicator. The cells on the lower side of the membrane were counted. Five random high-power fields $(\times 200)$ were selected and the mean number of cells was identified as the cell invasion number. The data represent at least three experiments performed in triplicate (mean \pm standard error).

\section{RNAi assay}

Renal carcinoma cell lines and primary cells were incubated in a six-well tissue culture dish without antibiotics for $24 \mathrm{~h}$ prior to transfection respectively, when they had reached $60-80 \%$ confluence. Negative control (NC), specific miR-21 inhibitor and 
mimic transfection reagent complexes were mixed with Lipofectamine 2000 (Invitrogen) according to the manufacturer's recommendations and then added to the cells. After $6 \mathrm{~h}$ at $37{ }^{\circ} \mathrm{C}$, the medium was changed and the cells were cultured in RPMI 1640 supplemented with 10\% heat-inactivated fetal bovine serum for various times. Silencing of miRNA21 was determined by qRT-PCR.

\section{Total RNA isolation and cDNA synthesis}

TRIzol reagent (CWbio. Co. Ltd., Beijing, China) was used to isolate total RNA from the cells. The isolated RNA was treated with DNase I (Invitrogen). The RNA concentration and purity were determined using a NanoDrop ${ }^{\circledR}$ ND-1000 (Nanodrop Products, Wilmington, DE, USA). The ratio of $28 \mathrm{~S} / 18 \mathrm{~S}$ was analyzed by Glyko Bandscan 5.0 (Glyko Biomedical Ltd., Novato, CA, USA). RNA quality and quantity were determined spectrophotometrically at 260 and $280 \mathrm{~nm}$, respectively. Reverse transcription of RNA was performed using the NCode miRNA FirstStrand cDNA Synthesis Kit (Invitrogen, Cat\#: MIRC-50).

\section{Primer design and quantitative real time PCR (qRT-PCR)}

For miRNA-21 the primer sequence was 5'-TAGCTTATCAGACTGATGTTGA-3' ${ }^{\prime}$ For U6, the sense primer sequence was $5^{\prime}$ CTCGCTTCGGCAGCACA- $3^{\prime}$ and the antisense primer sequence was $5^{\prime}$-AACGCTTCACGAATTTGCGT- ${ }^{\prime}$.

qRT-PCR was performed using the Light Cycler 2.0 RealTime PCR System (Roche, Basel, Switzerland) in a total volume of $20 \mu \mathrm{L}$ in glass capillaries containing $2 \mu \mathrm{L}$ of cDNA, $0.8 \mu \mathrm{L}$ of each primer, and $10 \mu \mathrm{L}$ of Light Cycler TaqMan Master Mix (Invitrogen, Cat\#: MIRC-50). The PCR for the miR-21 gene was initiated using a 10 min denaturation step at $95{ }^{\circ} \mathrm{C}$ followed by termination with a $30 \mathrm{~s}$ cooling step at $40{ }^{\circ} \mathrm{C}$. The cycling protocol consisted of denaturation at $95{ }^{\circ} \mathrm{C}$ for $15 \mathrm{~s}$ and annealing at $60{ }^{\circ} \mathrm{C}$ for $60 \mathrm{~s} ; 40$ cycles. Fluorescence detection was performed at the end of each extension step. An additional extension step of $5 \mathrm{~min}$ at $72{ }^{\circ} \mathrm{C}$ was added following the completion of 40 cycles. All PCR reactions were performed in duplicate. The PCR products were confirmed by melting curve analysis. We used the mathematical delta-delta method (ratio $=2^{-\Delta \Delta \mathrm{CT}}$ ) developed by PE Applied Biosystems (Perkin Elmer, Waltham, MA, USA) to compare relative expression results between treatments in qRT-PCR.

\section{Western blot}

To prepare whole cell extracts, cells at $90 \%$ confluence were washed in phosphate-buffered saline (PBS) before incubation with lysis buffer (1\% Triton X-100, $150 \mathrm{mM} \mathrm{NaCl,} 10 \mathrm{mM}$ Tris, pH 7.4, 1 mM EDTA, 1 mM EGTA, pH 8.0, $0.2 \mathrm{mM} \mathrm{Na}_{3} \mathrm{VO}_{4}$, $0.2 \mathrm{mM}$ phenylmethylsulfonyl fluoride, $0.5 \%$ Nonidet $\mathrm{P}-40$ ) on ice for $10 \mathrm{~min}$. The cell lysates were clarified by centrifugation at $12000 \mathrm{rpm}$ for $10 \mathrm{~min}$ and the supernatants were collected. The concentrations of total proteins were measured using a BCA Protein Assay Kit (Beyotime, Jiangsu, China). A total of $40 \mu \mathrm{g}$ protein was separated using SDS-PAGE and transferred onto PVDF membranes; the membranes were then blocked in 5\% fatfree milk at room temperature for $1 \mathrm{~h}$. After incubation with rabbit primary antibodies against PDCD4 (Abcam, Cambridge, UK) at a dilution of $1: 10000$ or GAPDH (Proteintech Group, Rosemont, IL, USA) at a dilution of $1: 4000$ at $4{ }^{\circ} \mathrm{C}$ overnight, the membranes were probed with goat anti-rabbit secondary antibodies at a dilution of $1: 4000$ at room temperature for $1 \mathrm{~h}$. The signals were detected using BeyoECL Plus (Beyotime) determined by quantitative analysis using UVP software. The ratio of $\mathrm{IOD}_{\mathrm{PDCD} 4} / \mathrm{IOD}_{\mathrm{GAPDH}}$ indicated the relative expression of PDCD4 protein.

\section{Statistical analysis}

SPSS 13.0 software (SPSS, Chicago, IL, USA) was employed for the analysis of all data. Data are expressed as the mean \pm standard deviation (SD), and Tamhane's T2 and analysis of variance (ANOVA) were used to determine the significance of the difference in multiple comparisons. An asterisk represents a $P$ value $<0.05$, which indicated a statistically significant result.

\section{Results}

\section{Microfluidic device and renal cell line invasion}

A microfluidic device developed in our previous study was used to investigate the lateral invasion of the renal cell lines and view the invasion process (Fig. 1A and B). The device is composed of a cell culture channel (red) and a stimulation channel (blue). The cell culture channel has both an outlet and an inlet while the stimulation channel has only inlet. The device is made of PDMS which is translucent. The device is sterilized and bonded to a $6 \mathrm{~cm}$ plate before use. The experimental design is shown in Fig. 1C. First, Matrigel ${ }^{\mathrm{TM}}$ was loaded into the stimulation

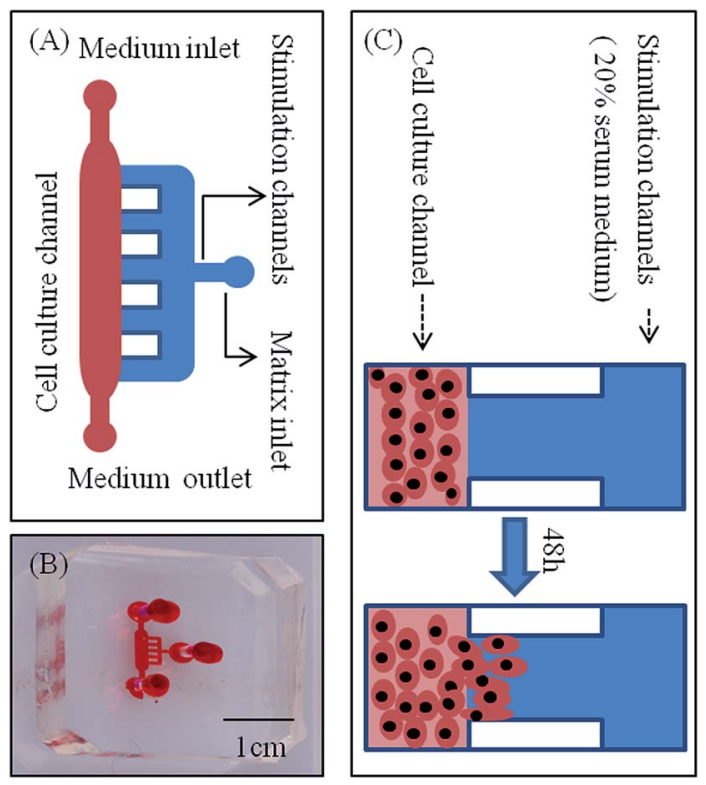

Fig. 1 Microfluidic device and renal cell line invasion. (A) Schematic representation of the microfluidic chip. The height of the main channel (red) and side channels (blue) is $150 \mu \mathrm{m}$ and $100 \mu \mathrm{m}$, respectively. (B) Photo of the microfluidic device filled with red ink. (C) Magnified illustration of the vessel unit, including one main channel, five side channels, and one matrix inlet. Scale bar $=20 \mu \mathrm{m}$. 
channels, then HK-2 or renal carcinoma cell lines (786-O, Caki-1 and A498) in serum-free medium were seeded into the cell culture channel, while medium containing $20 \%$ serum was loaded into the stimulation channel. Using this microfluidic device (Fig. 1A-C), both the HK-2 and renal carcinoma cell lines were cultured successfully. Using this system we found that only renal carcinoma cell lines invaded into the matrix with long cellular processes (Fig. 2A). The invasion area of 786-O $\left(21478.60 \pm 7168.20 \mu^{2}\right)$, Caki-1 $\left(22386.46 \pm 4447.32 \mu \mathrm{m}^{2}\right)$, and A498 (23706.67 $\left.\pm 6382.49 \mu^{2}\right)$ was significantly greater than that of HK-2 $\left(235.24 \pm 384.05 \mu \mathrm{m}^{2}\right)$. To evaluate invasion abilities of renal carcinoma cell lines and HK-2 further, we performed the Transwell ${ }^{\circledR}$ with Matrigel ${ }^{\mathrm{TM}}$ coating. And this revealed same results with microfluidic assay (Fig. 2B). Cell invasion number in pre view was used to evaluate cell invasion ability. The invasion ability of 786-O, Caki-1, A498 were higher than HK-2. These results suggest that renal carcinoma cell lines have distinct invasion abilities.

\section{Invasive ability of primary RCC cells}

To further investigate the invasive ability of RCC, four samples of primary cells were isolated from RCC, and named as C995, C147, C316 and C413 according to each patient's admission number. These primary cells were cultured successfully using our microfluidic device under the same culture conditions as renal carcinoma cell lines. Medium containing 20\% serum was then loaded into the stimulation channel, and $48 \mathrm{~h}$ later, the invasion area of primary cells and HK-2 cells was observed. The invasion areas of C147 (3881.56 $\left.\pm 1776.59 \mu \mathrm{m}^{2}\right), \mathrm{C} 413$ (7942.46 $\left.\pm 3769.38 \mu^{2}\right)$, C316 $\left(18999.70 \pm 5068.86 \mu \mathrm{m}^{2}\right)$ and C995 $\left(28196.62 \pm 13691.84 \mu \mathrm{m}^{2}\right)$ were found to be significantly higher than that of HK-2 $\left(235.24 \pm 384.05 \mu \mathrm{m}^{2}\right)$ (Fig. 3A). Although the invasion areas of C147 and C143 were not as high as C316 or C995, these results suggest that primary cells also have distinct invasive abilities.

\section{Expression of miR-21 in renal carcinoma cells and RNAi assay}

We next determined the expression levels of miR-21 in renal cancer cells (786-O, Caki-1, A498, C995, C147, C316 and C413) and in a normal renal cell line (HK-2) (Fig. 3B). In the renal cancer cell lines and in primary cells levels of endogenous miR21 were higher than HK-2 cells according to qRT-PCR analysis (Fig. 3B).

\section{Invasive ability of renal cell lines after altering expression of miR-21}

We cultured HK-2 cells and renal carcinoma cell lines that had been transfected with miR-21 mimic or miR-21 inhibitor in our
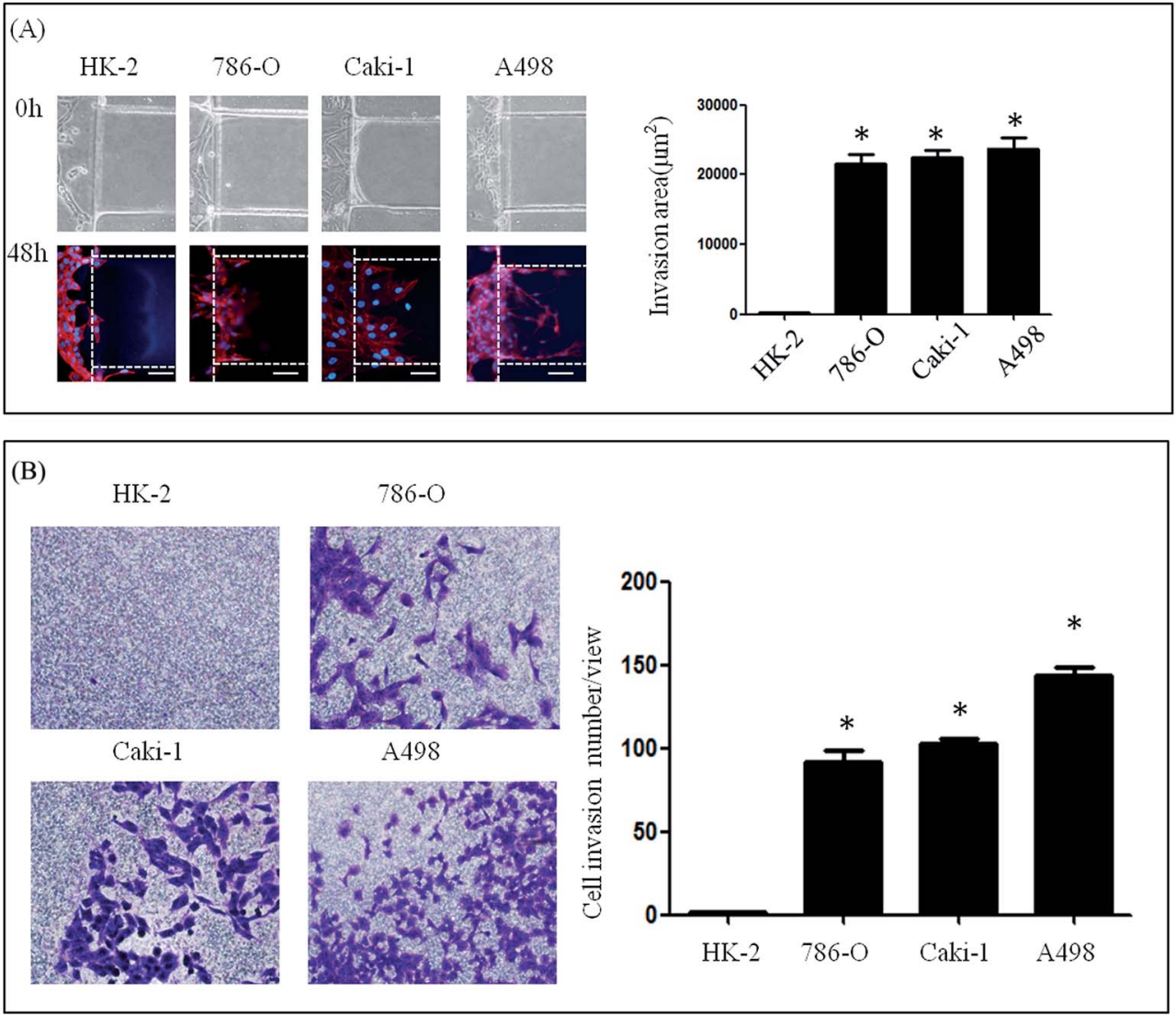

Fig. 2 Invasion of renal carcinoma cell lines. (A) Microfluidic assay. The invasion ability of 786-O, Caki-1 and A498 showed significantly higher compared to HK-2. (B) Transwell $®$ invasion assay. Significantly more 786-O, Caki-1 and A498 cells invaded through the matrix coating on the Transwell 1 membrane compared to HK-2. 

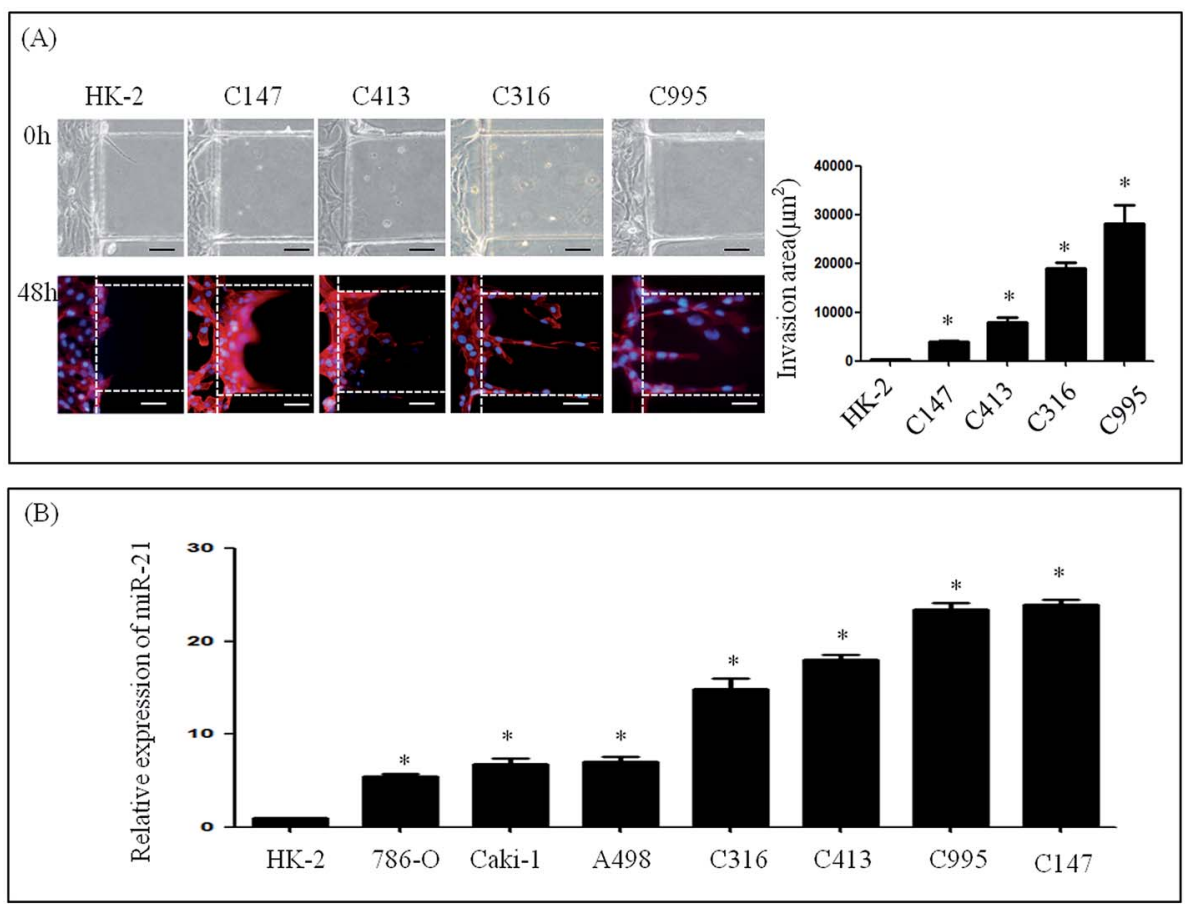

Fig. 3 Invasion of primary renal carcinoma cells and expression of miR-21. (A) Invasion of HK-2 and primary renal carcinoma cells. (B) Expression of miR-21 in HK-2, renal carcinoma cell lines and primary renal carcinoma cells.

microfluidic device and stimulated them with $20 \%$ serum for $48 \mathrm{~h}$. HK-2 cells transfected with miR-21 mimic showed high invasive abilities compared to mimic NC $\left(235.24 \pm 384.05 \mu \mathrm{m}^{2}\right.$ vs. $2502.66 \pm 1527.75 \mu \mathrm{m}^{2}$ ) (Fig. $4 \mathrm{~A}$ ). This result demonstrated that the invasion area of $786-\mathrm{O}$, Caki-1 and A498 cells treated with miR-21 inhibitor was significantly smaller than that of 786O Caki-1 and A498 cells treated with miR-21 inhibitor NC $\left(21478.60 \pm 7168.20 \mu \mathrm{m}^{2} v s .8603 .51 \pm 5461.17 \mu \mathrm{m}^{2}, 22386.46 \pm\right.$ $4447.32 \mu \mathrm{m}^{2}$ vs. $7942.46 \pm 3769.38 \mu \mathrm{m}^{2}, 23706.67 \pm 6382.49$ $\mu \mathrm{m}^{2}$ vs. $4949.94 \pm 3681.27 \mu \mathrm{m}^{2}$ ) (Fig. $4 \mathrm{~B}-\mathrm{D}$ ).

\section{Invasive ability of primary RCC cells after knocking down} miR-21

To test the involvement of miR-21 in the invasion of primary renal carcinoma cells (C147, C413, C316 and C995), we transfected them with miR-21 inhibitor and negative control siRNA. After stimulation with $20 \%$ serum for $48 \mathrm{~h}$, the invasion areas of C147 cells and C413 cells treated with miR-21 inhibitor were smaller compared with those treated with inhibitor $\mathrm{NC}\left(3881.56 \pm 1776.59 \mu \mathrm{m}^{2}\right.$ vs. $3189.62 \pm 2078.81 \mu \mathrm{m}^{2}, 7942.46$ $\pm 3769.38 \mu \mathrm{m}^{2}$ vs. $5935.06 \pm 4324.74 \mu \mathrm{m}^{2}$ ), but the difference was not statistically significant (Fig. 5A and B). Further, treatment of C316 cells and C995 cells with miR-21 inhibitor resulted in a smaller invasion area compared with treatment with inhibitor NC $\left(18999.70 \pm 5068.86 \mu \mathrm{m}^{2}\right.$ vs. $5188.44 \pm$ $3307.50 \mu \mathrm{m}^{2}, \quad 28196.62 \pm 13691.84 \mu \mathrm{m}^{2}$ vs. $9277.06 \pm$ $\left.3920.32 \mu \mathrm{m}^{2}\right)(P<0.05)$ (Fig. 5C and D). This result suggests that knockdown of the miR-21 gene could inhibit cell invasion by renal carcinoma.

\section{Discussion}

RCC is one of the most common urological cancers, and 20$30 \%$ of patients with RCC already have distant metastases with clinical symptoms upon diagnosis. For this reason, RCC, and especially its invasion and metastasis process or mechanism, is currently a hot topic of study. ${ }^{26-29}$ The Boyden chamber assay is widely used to study RCC invasion and metastasis but also has inherent limitations. This system lacks real-time observation and it is inherently difficult to directly assess tumor cell interaction with stromal cells. ${ }^{30}$ In addition, since cells move from the upper transwell to the lower culture chamber, cell invasion may be induced by gravity. ${ }^{31}$ For cell-based assays in a microfluidic device, one of the important advantages is to provide a well-controlled environment for precise study of cellular activities. ${ }^{32-34}$ Transendothelial invasion of tumor aggregates has been successfully observed in a microfluidic device, ${ }^{16}$ while another study used this microfluidic device to investigate whether HK-2 cells could migrate across the basement membrane following stimulation with transforming growth factor $\beta 1$ (TGF- $\beta 1$ ), C3a, or heat-inactivated healthy human serum (HHS).$^{35}$ However, to our knowledge, such a microfluidic device has rarely been used to observe RCC invasion in real time. In this study, we cultured HK-2, renal carcinoma cell lines and primary renal carcinoma cells using a microfluidic device. After stimulating with $20 \%$ serum for $48 \mathrm{~h}$ and observing the cells under a microscope, we found that both renal carcinoma cell lines and primary renal carcinoma cells showed higher invasive ability compared with HK-2 cells. Most studies of RCC have been carried out using renal carcinoma cell lines such as 

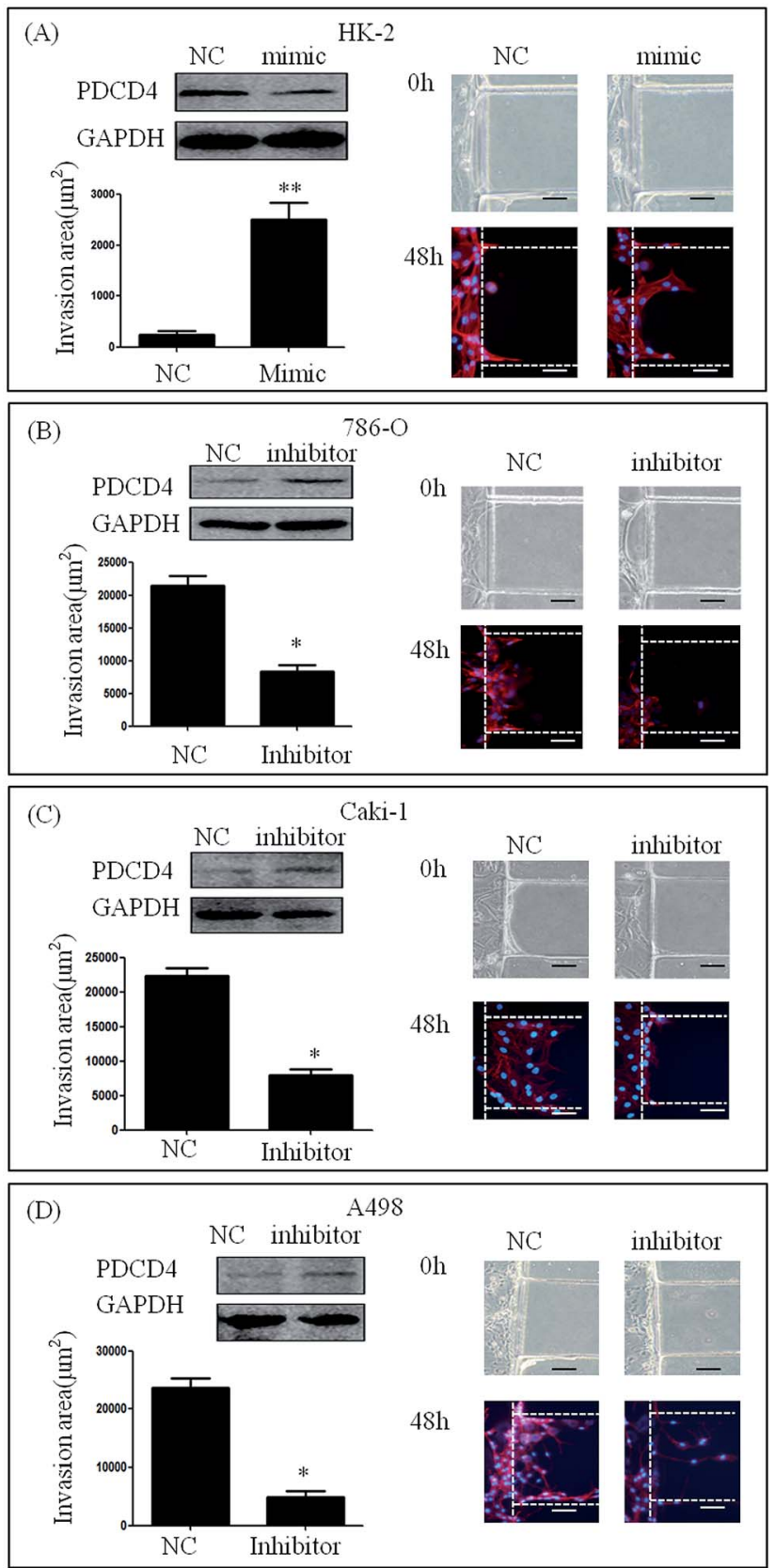

Fig. 4 Invasion in renal cancer cell lines after regulation of miR-21. Western blot assay was used to detect ectopic PDCD4 in these cell lines, as indicated in the upper panel, where GAPDH was used as a loading control. (A) The result of invasion assay of the HK-2 cells transfected with miR-21 mimic NC, or miR-21 mimic after $48 \mathrm{~h}$. The invasion area of $\mathrm{HK}-2$ cells treated with mimic were significantly higher than those of the cells treated with mimic NC. (B-D) The result of invasion assay for the renal cancer cell lines transfected with miR-21 inhibitor NC, or miR-21 inhibitor, measured at $48 \mathrm{~h}$. The invasion area of renal cancer cell lines treated with inhibitor NC was significantly higher than that of those treated with inhibitor. * indicates $P<0.05$. Scale bar $=20 \mu \mathrm{m}$.

A498, 786-O, or Caki-1 only. In this study, we used primary renal carcinoma cells and found that, compared with the effects observed in cell lines, the results obtained with primary cells were more persuasive. We concluded that we could use this
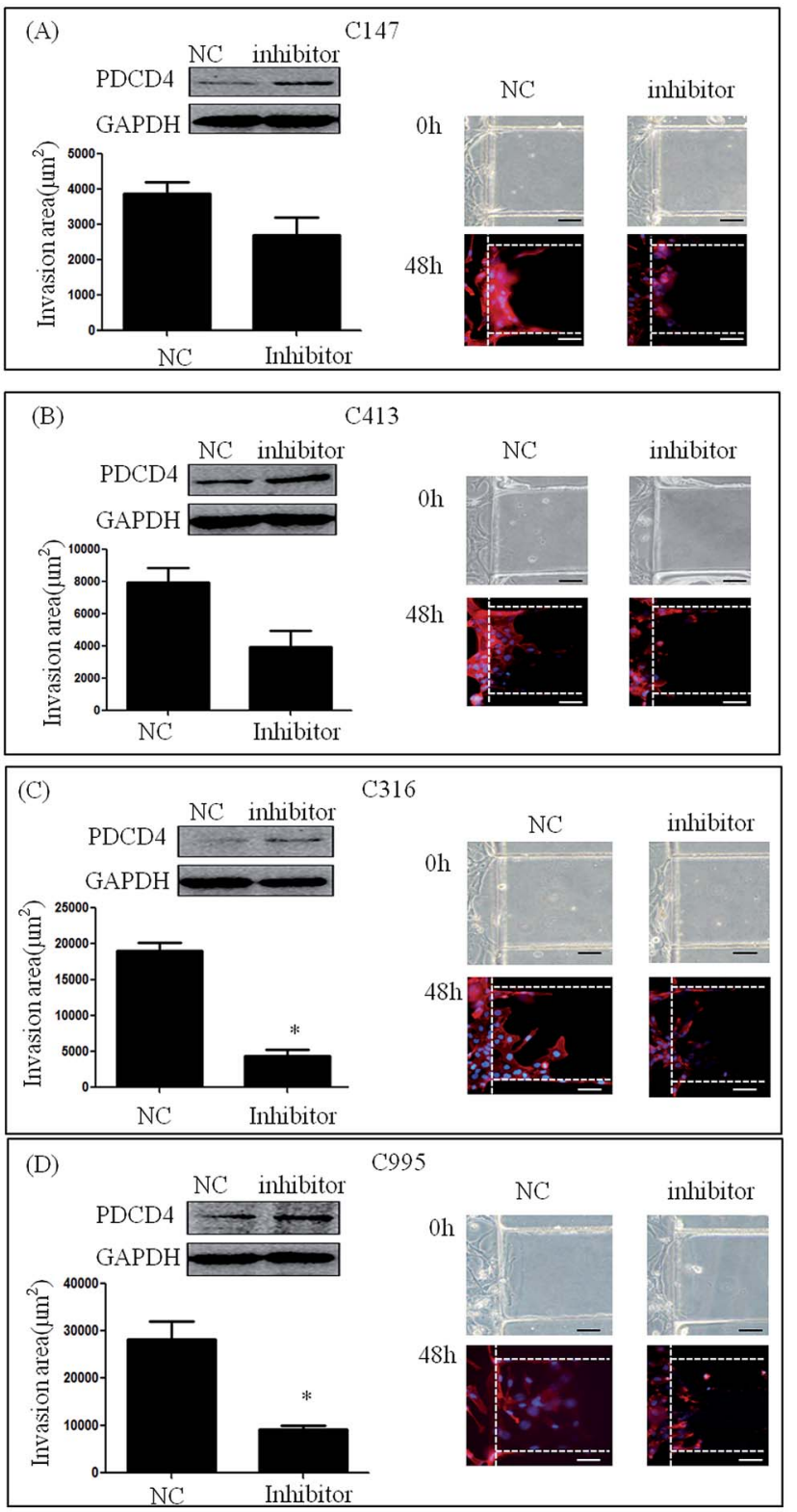

Fig. 5 Invasion in primary cells after treatment with miR-21 inhibitor. Western blot assay was used to detect ectopic PDCD4 expression in these cell lines, as indicated in the upper panel, where GAPDH was used as a loading control. (A and B) Invasion areas of C147 and C413 treated with miR-21 inhibitor were lower than those of cells treated with inhibitor NC. (C and D) Invasion areas of C316 and C995 treated with miR-21 inhibitor were significantly lower than those of cells treated with inhibitor NC.* indicates $P<0.05$. Scale bar $=20 \mu \mathrm{m}$.

microfluidic device to study factors such as miRs, drugs, and cytokines, that are more physiological factors influencing RCC invasion.

For RCC, miR-21 could be a promising, convenient and potentially non-invasive prognostic marker, and could allow monitoring of cancer progression or recurrence. ${ }^{36}$ In this study, we aimed to investigate whether miR-21 could be a therapeutic target. Our results showed that the invasion area of renal carcinoma cell lines treated with miR-21 inhibitor was 
significantly smaller than that of untreated renal carcinoma cell lines. This demonstrated that we could regulate the invasive ability of cells by controlling miR-21. Next, we attempted to control the miR-21 expression of primary renal carcinoma cells. The result showed that knockdown of miR-21 reduced the invasive ability of primary cells, implying that inhibition of miR21 expression could inhibit RCC invasion. To our knowledge, RCC is insensitive to chemotherapy and radiotherapy; therefore, effective post-operative adjuvant therapies are lacking. ${ }^{5}$ The combined traditional immune therapy using interleukin (IL)-II and interferon does not improve survival of patients with relapsed or advanced RCC, ${ }^{37}$ with five- and 10-year survival rates of $<10 \% .{ }^{38}$ At present, molecular-targeted therapy is used in post-operative adjuvant therapy for patients with early-stage RCC or as palliative treatment for patients with advanced RCC. ${ }^{39-42}$ Although the objective effective rate has evidently increased, the overall survival rate among RCC patients is low. ${ }^{6}$ In addition, molecular-targeted therapy is not only expensive but also causes many complications (hand-foot syndrome, sicchasia and emesia). ${ }^{41-43}$ As time goes by, drug resistance of RCC for molecular-targeted therapy is inevitable. Consequently it is extremely urgent to develop a new drug for RCC. The results of this study indicate that miR-21 could be a therapeutic target of RCC.

There are some molecular pathways of miR-21 which may play an important role in the process of RCC invasion, such as PDCD4, CDKN1A, and PTEN. ${ }^{23,43,44}$ In our study, we only broadly observed the influence of miR-21 on RCC invasion, without investigating which one or more molecular pathways could be the key of RCC therapy. Additionally, the number of primary renal carcinoma cell samples was only four, causing our study to lack conviction. We will still collect renal carcinoma cell samples in our further study. For C147 cells and C413 cells, the invasion areas of cells treated with miR-21 inhibitor were smaller compared with those treated with inhibitor NC, but the difference was not statistically significant. The cell line about renal cell carcinoma was taken from the tumor tissue of the patient, so tumor heterogeneity of the patients may lead to the non-aligned phenomenon. Moreover, post-transcriptional modification about the target genes of miR-21 may be also related to the phenomenon. All these are limitations of our study, but could be a direction of our future studies.

\section{Conclusions}

In summary, we cultured renal cell lines and primary renal carcinoma cells in vitro using our microfluidic device, and using this technique we reproduced RCC invasion at the cellular level. This device offers a novel platform which is a rapid and inexpensive way to study the mechanism of RCC invasion in vitro. We found that knocking down miR-21 could lead to a reduction in the invasive ability of renal carcinoma cells. These data suggest that miR-21 could be a new therapeutic target of RCC.

\section{Conflicts of interest}

The authors have declared that no competing interests exist.

\section{Acknowledgements}

The study was funded by the Natural Science Foundation of China (No. 81572505).

\section{References}

1 D. J. van Spronsen, P. F. Mulders and P. H. De Mulder, Novel treatments for metastatic renal cell carcinoma, Crit. Rev. Oncol. Hematol., 2005, 55(3), 177-191.

2 H. T. Cohen and F. J. McGovern, Renal-cell carcinoma, $N$. Engl. J. Med., 2005, 353(23), 2477-2490.

3 P. L. Crispen, R. H. Breau, C. Allmer, C. M. Lohse, J. C. Cheville, B. C. Leibovich, et al., Lymph node dissection at the time of radical nephrectomy for high-risk clear cell renal cell carcinoma: indications and recommendations for surgical templates, Eur. Urol., 2011, 59(1), 18-23.

4 Z. Wotschofsky, J. Liep, H. A. Meyer, M. Jung, I. Wagner, A. C. Disch, et al., Identification of metastamirs as metastasis-associated microRNAs in clear cell renal cell carcinomas, Int. J. Biol. Sci., 2012, 8(10), 1363-1374.

5 B. Escudier, Advanced renal cell carcinoma: current and emerging management strategies, Drugs, 2007, 67(9), 1257-1264.

6 T. Cheng, L. Wang, Y. Li, C. Huang, L. Zeng and J. Yang, Differential microRNA expression in renal cell carcinoma, Oncol. Lett., 2013, 6(3), 769-776.

7 J. El-Ali, P. K. Sorger and K. F. Jensen, Cells on chips, Nature, 2006, 442(7101), 403-411.

8 G. M. Whitesides, The origins and the future of microfluidics, Nature, 2006, 442(7101), 368-373.

9 T. Liu, C. Li, H. Li, S. Zeng, J. Qin and B. Lin, A microfluidic device for characterizing the invasion of cancer cells in 3-D matrix, Electrophoresis, 2009, 30(24), 4285-4291.

10 T. Liu, B. Lin and J. Qin, Carcinoma-associated fibroblasts promoted tumor spheroid invasion on a microfluidic 3D co-culture device, Lab Chip, 2010, 10(13), 1671-1677.

11 J. M. Peyrin, B. Deleglise, L. Saias, M. Vignes, P. Gougis, S. Magnifico, et al., Axon diodes for the reconstruction of oriented neuronal networks in microfluidic chambers, $L a b$ Chip, 2011, 11(21), 3663-3673.

12 S. R. Khetani and S. N. Bhatia, Microscale culture of human liver cells for drug development, Nat. Biotechnol., 2008, 26(1), 120-126.

13 D. Huh, B. D. Matthews, A. Mammoto, M. Montoya-Zavala, H. Y. Hsin and D. E. Ingber, Reconstituting organ-level lung functions on a chip, Science, 2010, 328(5986), 1662-1668.

14 K. E. Sung, N. Yang, C. Pehlke, P. J. Keely, K. W. Eliceiri, A. Friedl, et al., Transition to invasion in breast cancer: a microfluidic in vitro model enables examination of spatial and temporal effects, Integr. Biol., 2011, 3(4), 439-450.

15 K. C. Chaw, M. Manimaran, F. E. Tay and S. Swaminathan, Matrigel coated polydimethylsiloxane based microfluidic devices for studying metastatic and non-metastatic cancer cell invasion and migration, Biomed. Microdevices, 2007, 9(4), 597-602.

16 Q. Zhang, T. Liu and J. Qin, A microfluidic-based device for study of transendothelial invasion of tumor aggregates in realtime, Lab Chip, 2012, 12(16), 2837-2842. 
17 M. Li, Y. Wang, Y. Song, R. Bu, B. Yin, X. Fei, et al., MicroRNAs in renal cell carcinoma: a systematic review of clinical implications (Review), Oncol. Rep., 2015, 33(4), 1571-1578.

18 S. D. Selcuklu, M. T. Donoghue and C. Spillane, miR-21 as a key regulator of oncogenic processes, Biochem. Soc. Trans., 2009, 37(Pt 4), 918-925.

19 X. Lu, Y. Liu, F. Luo, A. Zhang, X. Liu, L. Lu, et al., MicroRNA21 activation of Akt via PTEN is involved in the epithelialmesenchymal transition and malignant transformation of human keratinocytes induced by arsenite, Toxicol. Res., 2016, 5(4), 1140-1147.

20 J. Li, S. Tan, R. Kooger, C. Zhang and Y. Zhang, MicroRNAs as novel biological targets for detection and regulation, Chem. Soc. Rev., 2014, 43(2), 506-517.

21 Z. A. Dotan, E. Fridman, Y. Spector, I. Barshack, A. Chajut, S. Rosenwald, et al., MicroRNAs as prognostic markers for survival in renal cell carcinoma conventional type T 2-4, J. Clin. Oncol., 2011, 29(suppl. 15), e21115.

22 N. Dey, F. Das, N. Ghoshchoudhury, C. C. Mandal, D. J. Parekh, K. Block, et al., microRNA-21 governs TORC1 activation in renal cancer cell proliferation and invasion, PLoS One, 2012, 7(6), 193-203.

23 L. Gu, H. Li, L. Chen, X. Ma, Y. Gao, X. Li, et al., MicroRNAs as prognostic molecular signatures in renal cell carcinoma: a systematic review and meta-analysis, Oncotarget, 2015, 6(32), 32545-32560.

24 X. Li, S. Xin, Z. He, X. Che, J. Wang, X. Xiao, et al., MicroRNA21 (miR-21) post-transcriptionally downregulates tumor suppressor PDCD4 and promotes cell transformation, proliferation, and metastasis in renal cell carcinoma, Cell. Physiol. Biochem., 2014, 33(6), 1631-1642.

25 Y. Sakurai, K. Kajimoto and H. Harashima, Anti-angiogenic nanotherapy via active targeting systems to tumors and adipose tissue vasculature, Biomater. Sci., 2015, 3(9), 12531265.

26 M. Ishida, S. Mikami, T. Shinojima, T. Kosaka, R. Mizuno, E. Kikuchi, et al., Activation of aryl hydrocarbon receptor promotes invasion of clear cell renal cell carcinoma and is associated with poor prognosis and cigarette smoke, Int. J. Cancer, 2015, 137(2), 299-310.

27 S. Mikami, M. Oya, R. Mizuno, T. Kosaka, K. Katsube and Y. Okada, Invasion and metastasis of renal cell carcinoma, Med. Mol. Morphol., 2014, 47(2), 63-67.

28 Y. Tomita, Treatment strategies for advanced renal cell carcinoma: A new paradigm for surgical treatment, Int. J. Urol., 2016, 23(1), 13-21.

29 R. Nishikawa, Y. Goto, S. Sakamoto, T. Chiyomaru, H. Enokida, S. Kojima, et al., Tumor-suppressive microRNA-218 inhibits cancer cell migration and invasion via targeting of LASP1 in prostate cancer, Cancer Sci., 2014, 105(7), 802-811.

30 T. Yu, Z. Guo, H. Fan, J. Song, Y. Liu, Z. Gao, et al., Cancerassociated fibroblasts promote non-small cell lung cancer cell invasion by upregulation of glucose-regulated protein 78 (GRP78) expression in an integrated bionic microfluidic device, Oncotarget, 2016, 7(18), 25593-25603.
31 K. F. Lei, H. P. Tseng, C. Y. Lee and N. M. Tsang, Quantitative study of cell invasion process under extracellular stimulation of cytokine in a microfluidic device, Sci. Rep., 2016, 6, 25557.

32 S. Halldorsson, E. Lucumi, R. Gomez-Sjoberg and R. M. Fleming, Advantages and challenges of microfluidic cell culture in polydimethylsiloxane devices, Biosens. Bioelectron., 2015, 63, 218-231.

33 M. Mehling and S. Tay, Microfluidic cell culture, Curr. Opin. Biotechnol., 2014, 25, 95-102.

34 C. W. Shields, C. D. Reyes and G. P. Lopez, Microfluidic cell sorting: a review of the advances in the separation of cells from debulking to rare cell isolation, Lab Chip, 2015, 15(5), 1230-1249.

35 M. Zhou, H. Ma, H. Lin and J. Qin, Induction of epithelial-tomesenchymal transition in proximal tubular epithelial cells on microfluidic devices, Biomaterials, 2014, 35(5), 13901401.

36 L. Gu, H. Li, L. Chen, X. Ma, Y. Gao, X. Li, et al., MicroRNAs as prognostic molecular signatures in renal cell carcinoma: a systematic review and meta-analysis, Oncotarget, 2015, 6(32), 32545-32560.

37 E. L. Broghammer and T. L. Ratliff, Immunotherapy of urologic tumors: principles and progress, Urol. Oncol., 2002, 7(2), 45-56.

38 P. Mulders, R. Figlin, J. B. deKernion, R. Wiltrout, M. Linehan, D. Parkinson, et al., Renal cell carcinoma: recent progress and future directions, Cancer Res., 1997, 57(22), 5189-5195.

39 B. Escudier, A. Pluzanska, P. Koralewski, A. Ravaud, S. Bracarda, C. Szczylik, et al., Bevacizumab plus interferon alfa-2a for treatment of metastatic renal cell carcinoma: a randomised, double-blind phase III trial, Lancet, 2007, 370(9605), 2103-2111.

40 B. Escudier, T. Eisen, W. M. Stadler, C. Szczylik, S. Oudard, M. Staehler, et al., Sorafenib for treatment of renal cell carcinoma: final efficacy and safety results of the phase III treatment approaches in renal cancer global evaluation trial, J. Clin. Oncol., 2009, 27(20), 3312-3318.

41 C. N. Sternberg, I. D. Davis, J. Mardiak, C. Szczylik, E. Lee, J. Wagstaff, et al., Pazopanib in locally advanced or metastatic renal cell carcinoma: results of a randomized phase III trial, J. Clin. Oncol., 2010, 28(6), 1061-1068.

42 G. Hudes, M. Carducci, P. Tomczak, J. Dutcher, R. Figlin, A. Kapoor, et al., Temsirolimus, interferon alfa, or both for advanced renal-cell carcinoma, N. Engl. J. Med., 2007, 356(22), 2271-2281.

43 M. Santoni, A. Conti, U. De Giorgi, R. Iacovelli, F. Pantano, L. Burattini, et al., Risk of gastrointestinal events with sorafenib, sunitinib and pazopanib in patients with solid tumors: a systematic review and meta-analysis of clinical trials, Int. J. Cancer, 2014, 135(4), 763-773.

44 M. S. Zaman, V. Shahryari, G. Deng, S. Thamminana, S. Saini, S. Majid, et al., Up-regulation of microRNA-21 correlates with lower kidney cancer survival, PLoS One, 2012, 7(2), e31060. 